Pacific Journal of Mathematics

CONVEX AND CONCAVE FUNCTIONS OF SINGULAR VALUES 


\title{
CONVEX AND CONCAVE FUNCTIONS OF SINGULAR VALUES OF MATRIX SUMS
}

\author{
R. C. ThOMPSON
}

A new set of inequalities for functions of singular values of matrix sums is established. These inequalities are complementary to a number of classically known inequalities in that the direction of the inequality sign is reversed. A matrix valued triangle inequality is also given. Special cases of these results are due to S. Yu. Rotfel'd.

1. Introduction. Let $A, B, C=A+B$ be $n$-square matrices with singular values $\alpha_{1} \geqq \cdots \geqq \alpha_{n}, \beta_{1} \geqq \cdots \geqq \beta_{n}, \gamma_{1} \geqq \cdots \geqq \gamma_{n}$ respectively. In [5] S. Yu. Rotfel'd stated and in [6] proved that

$$
f\left(\gamma_{1}\right)+\cdots+f\left(\gamma_{n}\right) \leqq f\left(\alpha_{1}\right)+\cdots+f\left(\alpha_{n}\right)+f\left(\dot{\beta_{1}}\right)+\cdots+f\left(\beta_{n}\right)
$$

when $f$ is an increasing concave function of a nonnegative real variable, with $f(0)=0$. This inequality is of some interest as in previously published work a convexity (rather than concavity) hypothesis has usually been necessary to establish results of the general type of (1). See, for example, Gohberg and Krein [3], page 49, or Marcus and Minc [4], pages 103 and 116. In this paper we shall uncover the algebraic foundation of (1) by giving a short proof of a generalization. Our proof, which is simpler and more direct than the proof of (1) given by Rotfel'd, will be based on an interesting matrix valued triangle inequality, a special case of which was given by Rotfel'd. We note that the methods used by Rotfel'd are very much adapted to the inequality (1) that he wished to prove, and do not appear capable of proving the extensions of his results to be presented below.

2. Positive semidefinite matrices. In this section we discuss an important special case for which sharper results are possible. Throughout $\$ 2$ we let $A, B, C=A+B$ be $n \times n$ Hermitian positive semidefinite matrices with eigenvalues $\alpha_{1} \geqq \cdots \geqq \alpha_{n}, \beta_{1} \geqq \cdots \geqq$ $\beta_{n}, \gamma_{1} \geqq \cdots \geqq \gamma_{n}$, respectively. Let $F\left(x_{1}, \cdots, x_{2 n}\right)$ and $G\left(x_{1}, \cdots, x_{2 n}\right)$ be functions of $2 n$ nonnegative variables, symmetric in these variables (i.e., remaining unchanged if the variables are permuted), with $F$ concave and $G$ convex: for all nonnegative vectors $x, y$ and real numbers $\theta$ with $0 \leqq \theta \leqq 1$,

$$
\begin{aligned}
& F(\theta x+(1-\theta) y) \geqq \theta F(x)+(1-\theta) F(y), \\
& G(\theta x+(1-\theta) y) \leqq \theta G(x)+(1-\theta) G(y) .
\end{aligned}
$$


Then we have:

Theorem 1. For Hermitian positive semidefinite $A, B, C=A+B$ and functions $F, G$ as above, the following inequalities hold:

$$
F\left(\gamma_{1}, \cdots, \gamma_{n}, 0, \cdots, 0\right) \leqq F\left(\alpha_{1}, \cdots, \alpha_{n}, \beta_{1}, \cdots, \beta_{n}\right)
$$

$G\left(\gamma_{1}, \cdots, \gamma_{n}, 0, \cdots, 0\right) \geqq G\left(\alpha_{1}, \cdots, \alpha_{n}, \beta_{1}, \cdots, \beta_{n}\right)$

$F\left(\frac{\gamma_{1}+\cdots+\gamma_{n}}{2 n}, \cdots, \frac{\gamma_{1}+\cdots+\gamma_{n}}{2 n}\right) \geqq F\left(\alpha_{1}, \cdots, \alpha_{n}, \beta_{1}, \cdots, \beta_{n}\right)$,

$G\left(\frac{\gamma_{1}+\cdots+\gamma_{n}}{2 n}, \cdots, \frac{\gamma_{1}+\cdots+\gamma_{n}}{2 n}\right) \leqq G\left(\alpha_{1}, \cdots, \alpha_{n}, \beta_{1}, \cdots, \beta_{n}\right)$

Proof. Since $A$ and $B$ are semidefinite, we may write $A=A_{1} A_{1}^{*}$, $B=B_{1} B_{1}^{*}$, where ${ }^{*}$ denotes adjoint. Then we have

$$
C=A_{1} A_{1}^{*}+B_{1} B_{1}^{*}=\left[A_{1}, B_{1}\right]\left[\begin{array}{l}
A_{1}^{*} \\
B_{1}^{*}
\end{array}\right]
$$

Reversing the order of the factors in the last product, and using the fact that this reversal leaves unchanged the nonzero eigenvalues, we see that

$$
\left[\begin{array}{c}
A_{1}^{*} \\
B_{1}^{*}
\end{array}\right]\left[A_{1}, B_{1}\right]=\left[\begin{array}{ll}
A_{1}^{*} A_{1} & A_{1}^{*} B_{1} \\
B_{1}^{*} A_{1} & B_{1}^{*} B_{1}
\end{array}\right]
$$

has $\gamma_{1}, \cdots, \gamma_{n}, 0, \cdots, 0$ as its eigenvalues. Making a block diagonal unitary similarity to diagonalize the blocks $A_{1}^{*} A_{1}$ and $B_{1} B_{1}^{*}$ in (3), we next see that $\alpha_{1}, \cdots, \alpha_{n}, \beta_{1}, \cdots, \beta_{n}$ are diagonal elements of a Hermitian matrix having $\gamma_{1}, \cdots, \gamma_{n}, 0, \cdots, 0$ as its eigenvalues. Consequently

$$
\left[\begin{array}{c}
\alpha_{1} \\
\cdot \\
\cdot \\
\cdot \\
\alpha_{n} \\
\beta_{1} \\
\cdot \\
\cdot \\
\cdot \\
\beta_{n}
\end{array}\right]=S\left[\begin{array}{c}
\gamma_{1} \\
\cdot \\
\cdot \\
\cdot \\
\gamma_{n} \\
0 \\
\cdot \\
\cdot \\
\cdot \\
0
\end{array}\right]
$$

where $S$ is a $2 n$-square doubly stochastic matrix. By Birkhoff's theorem this implies that the left hand side of (4) belongs to the convex hull of all 
rearrangements of the column vector $v=\left[\gamma_{1}, \cdots, \gamma_{n}, 0, \cdots, 0\right]^{\mathrm{T}}$. Now it is well known that a symmetric concave function $F$ on the convex polytope spanned by all rearrangements of a fixed vector $v$ assumes its least value at the vector $v$ and its greatest value at the vector $\bar{v}$ in which each component is the average of the components of $v$. See, for example, [4; p. 104]. (The argument there only shows that $F$ achieves its maximum at a vector in the convex polytope having all components equal; however, it is easy to show that $\bar{v}$ is the unique such vector.) This proves the first and third inequalities of the theorem; the second and fourth follow from the corresponding fact for convex functions $G$ in which the words "least" and "greatest" above are interchanged.

Previously known inequalities for functions on the eigenvalues of $A$, $B, C=A+B$ take the form

$$
\begin{aligned}
& F_{1}\left(\gamma_{1}, \cdots, \gamma_{n}\right) \geqq F_{1}\left(\alpha_{1}+\beta_{1}, \cdots, \alpha_{n}+\beta_{n}\right), \\
& G_{1}\left(\gamma_{1}, \cdots, \gamma_{n}\right) \leqq G_{1}\left(\alpha_{1}+\beta_{1}, \cdots, \alpha_{n}+\beta_{n}\right), \\
& F_{1}\left(\gamma_{1}, \cdots, \gamma_{n}\right) \leqq F_{1}\left(\frac{\alpha_{1}+\beta_{1}+\cdots+\alpha_{n}+\beta_{n}}{n}, \cdots\right. \\
& \left.\frac{\alpha_{1}+\beta_{1}+\cdots+\alpha_{n}+\beta_{n}}{n}\right) \\
& G_{1}\left(\gamma_{1}, \cdots, \gamma_{n}\right) \geqq G_{1}\left(\frac{\alpha_{1}+\beta_{1}+\cdots+\alpha_{n}+\beta_{n}}{n}, \cdots\right. \\
& \left.\frac{\alpha_{1}+\beta_{1}+\cdots+\alpha_{n}+\beta_{n}}{n}\right)
\end{aligned}
$$

where $F_{1}\left(x_{1}, \cdots, x_{n}\right)$ and $G_{1}\left(x_{1}, \cdots, x_{n}\right)$ are symmetric functions of $n$ nonnegative variables, with $F_{1}$ concave and $G_{1}$ convex. These follow from the well known results that

$$
\gamma_{1}+\cdots+\gamma_{k} \leqq \alpha_{1}+\cdots+\alpha_{k}+\beta_{1}+\cdots+\beta_{k} \text { for } k=1, \cdots, n,
$$

(with equality for $k=n$ ), and therefore

$$
\left[\begin{array}{c}
\gamma_{1} \\
\cdot \\
\cdot \\
\gamma_{n}
\end{array}\right]=S_{1}\left[\begin{array}{c}
\alpha_{1}+\beta_{1} \\
\cdot \\
\cdot \\
\cdot \\
\alpha_{n}+\beta_{n}
\end{array}\right]
$$

for some $n$-square doubly stochastic matrix $S_{1}$. Evidently (2.1) and (2.2) 
may be regarded as inequalities complementary to the previously known inequalities (5.1) and (5.2).

In the special case $F\left(x_{1}, \cdots, x_{2 n}\right)=f\left(x_{1}\right)+\cdots+f\left(x_{2 n}\right)$, with $f(x)$ concave and $f(0)=0$, inequality (2.1) reduces to the inequality (1) found by Rotfel'd.

The device of proving matrix inequalities by reversing the order of the factors, as in (3), was first introduced by Wielandt, in another context, and appears in his lecture notes [9].

3. A triangle inequality for matrices. If $A$ is a square matrix, not necessarily Hermitian, define

$$
|A|=\left(A A^{*}\right)^{1 / 2},
$$

where the positive semidefinite determination of the square root is used. If $H$ and $K$ are Hermitian matrices, the notation $H \leqq K$ will signify that $K-H$ is positive semidefinite.

THEOREM 2. Let $A$ and $B$ be square matrices, not necessarily Hermitian. Then unitary matrices $U$ and $V$ exist such that

$$
|A+B| \leqq U|A| U^{*}+V|B| V^{*}
$$

REMARK 1. Without the presence of the unitary matrices $U$ and $V$, this result would be false.

Remark 2. For the special case of Hermitian $A$ and $B$, with rank $B=1$, this result appears in Rotfel'd's paper [6]. The method used by Rotfel'd can, after some simplification, be used to obtain (6) for arbitrary Hermitian $A$ and $B$, but does not immediately extend to the nonHermitian case.

Proof. We first present a lemma giving a result of Fan and Hoffman [2], reproved in [1], and reproved again in [7]. For completeness we include the short proof from [7].

Lemma. Let $M$ be a matrix, not necessarily Hermitian, and denote the eigenvalues of $\frac{1}{2}\left(M+M^{*}\right)$ and $\left(M M^{*}\right)^{1 / 2}$ respectively by $\lambda_{1} \geqq \cdots \geqq \lambda_{n}$, $s_{1} \geqq \cdots \geqq s_{n}$. Then

$$
\lambda_{\imath} \leqq s_{t} \quad \text { for } i=1, \cdots, n .
$$

Proof of lemma. Take $v_{1}, \cdots, v_{n}$ and $w_{1}, \cdots, w_{n}$ to be orthonormal sets of eigenvectors for $\frac{1}{2}\left(M+M^{*}\right)$ and $M M^{*}$ respectively: 


$$
\frac{1}{2}\left(M+M^{*}\right) v_{i}=\lambda_{\imath} v_{l}, \quad M M^{*} w_{\imath}=s_{\imath}^{2} w_{i}, \quad i=1, \cdots, n .
$$

Let $k$ be fixed, $1 \leqq k \leqq n$. The spans of $v_{1}, \cdots, v_{k}$ and $w_{k}, \cdots, w_{n}$ intersect in at least one dimension; let $x$ be a unit vector in this intersection. Then

$$
\left(\frac{1}{2}\left(M+M^{*}\right) x, x\right) \geqq \lambda_{k}, \quad\left(M M^{*} x, x\right) \leqq s_{k}^{2},
$$

where $($, ) denotes inner product. We now have:

$$
\begin{aligned}
\lambda_{k} \leqq\left(\frac{1}{2}\left(M+M^{*}\right) x, x\right) & =\operatorname{Re}\left(M^{*} x, x\right) \leqq\left|\left(M^{*} x, x\right)\right| \\
& \leqq\left\|M^{*} x\right\|=\left(M M^{*} x, x\right)^{1 / 2} \leqq s_{k} .
\end{aligned}
$$

This completes the proof of the lemma.

Turning to the proof of Theorem 2, let $C=A+B$. Passing to $C W=A W+B W$ with $W$ unitary, we see that no generality is lost if we take $C$ to be positive semidefinite. From $C=A+B$ we then obtain, after forming the adjoint and adding, the equation

$$
C=\frac{1}{2}\left(A+A^{*}\right)+\frac{1}{2}\left(B+B^{*}\right) .
$$

We claim: $\frac{1}{2}\left(A+A^{*}\right) \leqq U|A| U^{*}$ for some unitary $U$. Indeed, take $U$ to be such that a unitary similarity simultaneously brings $\frac{1}{2}\left(A+A^{*}\right)$ and $U|A| U^{*}=U\left(A A^{*}\right)^{1 / 2} U^{*}$ into diagonal form with diagonal elements in nonincreasing order. By the lemma, each eigenvalue of $\frac{1}{2}\left(A+A^{*}\right)$ is dominated by the corresponding eigenvalue of $U|A| U^{*}$. This means:

$$
\frac{1}{2}\left(A+A^{*}\right) \leqq U|A| U^{*} .
$$

In the same way unitary $V$ exists such that $\frac{1}{2}(B+B)^{*} \leqq V|B| V^{*}$. Substituting these inequalities into (7) and using $C=|C|$, we obtain (6).

This lemma may be used to give a variant of certain proofs in [8].

4. Singular value inequalities. We continue to let $F\left(x_{1}, \cdots, x_{2 n}\right)$ and $G\left(x_{1}, \cdots, x_{2 n}\right)$ be symmetric functions of $2 n$ nonnegative variables, with $F$ concave and $G$ convex. We now let $A, B$, $C=A+B$ be not necessarily Hermitian matrices with singular values $\alpha_{1} \geqq \cdots \geqq \alpha_{n}, \beta_{1} \geqq \cdots \geqq \beta_{n}$ and $\gamma_{1} \geqq \cdots \geqq \gamma_{n}$ respectively.

THEOREM 3. Let arbitrary matrices $A, B, C=A+B$ and symmetric functions $F, G$ be as described above. Then:

(i) inequality (2.1) holds if $F$ is nondecreasing as well as concave,

(ii) inequality (2.2) holds if $G$ is nonincreasing as well as convex, 
(iii) inequality (2.3) holds if $F$ is nonincreasing as well as concave,

(iv) inequality (2.4) holds if $G$ is nondecreasing as well as convex.

Here, for example, $F$ nondecreasing means that $F\left(x_{1}, \cdots, x_{2 n}\right)$ is a nondecreasing function of $x_{1}$ for fixed $x_{2}, x_{3}, \cdots, x_{2 n}$.

Proof. It is well known that if $H, K$ are Hermitian matrices with $H \leqq K$ then the eigenvalues $h_{1} \geqq \cdots \geqq h_{n}$ and $k_{1} \geqq \cdots \geqq k_{n}$ of $H$ and $K$ satisfy $h_{i} \leqq k_{\imath}$ for each $i$. Indeed, set $K=H+P$, with $P$ positive semidefinite, and use the easily proved inequality $k_{i} \geqq h_{i}+p_{n}$, where $p_{n}$ is the smallest eigenvalue of $P$. From $C=A+B$ we get

$$
|C| \leqq U|A| U^{*}+V|B| V^{*}
$$

thus the singular values $\gamma_{1} \geqq \cdots \geqq \gamma_{n}$ of $C$ are dominated, term by term, by the singular values $\gamma_{1}^{\prime} \geqq \cdots \geqq \gamma_{n}^{\prime}$ of $U|A| U^{*}+V|B| V^{*}$. Using first the nondecreasing nature of $F$, then Theorem 1 applied to $U|A| U^{*}$ and $V|B| V^{*}$, we get

$$
F\left(\gamma_{1}, \cdots, \gamma_{n}, 0, \cdots, 0\right) \leqq F\left(\gamma_{1}^{\prime}, \cdots, \gamma_{n}^{\prime}, 0, \cdots, 0\right) \leqq F\left(\alpha_{1}, \cdots, \alpha_{n}, \beta_{1}, \cdots, \beta_{n}\right)
$$

The remaining parts of the theorem are proved similarly.

\section{REFERENCES}

1. Ky Fan, On strictly dissipative matrices, Linear Algebra and Appl., 9 (1974), 233-241.

2. Ky Fan and A. J. Hoffman, Some metric inequalities in the space of matrices, Proc. Amer. Math. Soc., 6 (1955), 111-116.

3. I. C. Gohberg and M. G. Krein, Introduction to the theory of linear nonselfadjoint operators, vol. 18, Translations of Mathematical Monographs, American Mathematical Society, Providence, R.I.

4. M. Marcus and H. Minc, A Survey of Matrix Theory and Matrix Inequalities, Allyn and Bacon, Boston.

5. S. Yu. Rotfel'd, Remarks on the singular numbers of a sum of completely continuous operators, Functional Anal. Appl., 1 (1967), 252-253.

6. S. Yu. Rotfel'd, The singular numbers of the sum of completely continuous operators, Topics in Mathematical Physics, vol. 3, Spectral Theory, edited by M. S. Berman, (1969), 73-78. English version published by Consultants Bureau, New York.

7. R. C. Thompson, Dissipative matrices and related results, Linear Algebra and Appl., 11 (1975), 155-169.

8. R. C. Thompson, Singular value inequalities for matrix sums and minus, Linear Algebra and Appl., 11 (1975), 251-269.

9. H. Wielandt, Topics in Analytic Theory of Matrices, lecture notes prepared by R. Meyer, University of Wisconsin, 1967.

Received February 6, 1976. The preparation of this paper was supported in part by Grant 72-2164 of the U.S. Airforce Scientific Research Office. 



\section{Pacific Journal of Mathematics}

\section{Vol. 66, No. $1 \quad$ November, 1976}

Helen Elizabeth. Adams, Factorization-prime ideals in integral domains ............ Patrick Robert Ahern and Robert Bruce Schneider, The boundary behavior of Henkin's kernel.

Daniel D. Anderson, Jacob R. Matijevic and Warren Douglas Nichols, The Krull

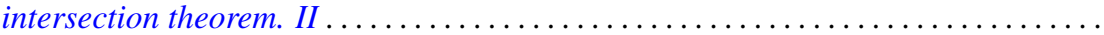

Efraim Pacillas Armendariz, On semiprime P.I.-algebras over commutative regular

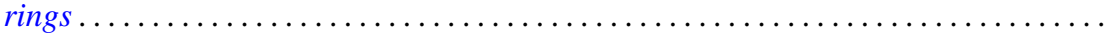

Robert H. Bird and Charles John Parry, Integral bases for bicyclic biquadratic fields

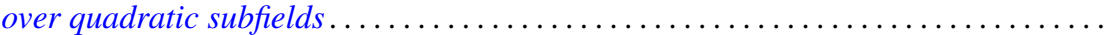

Tae Ho Choe and Young Hee Hong, Extensions of completely regular ordered

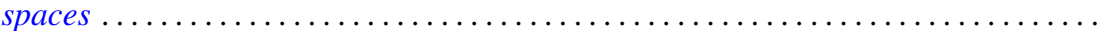

John Dauns, Generalized monoform and quasi injective modules ...............

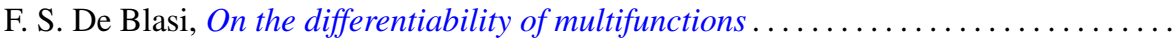

Paul M. Eakin, Jr. and Avinash Madhav Sathaye, R-endomorphisms of $R[[X]]$ are

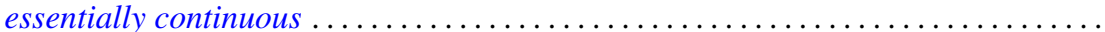

Larry Quin Eifler, Open mapping theorems for probability measures on metric

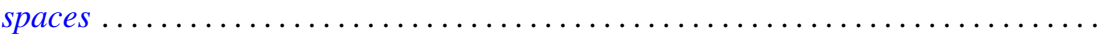

Garret J. Etgen and James Pawlowski, Oscillation criteria for second order self adjoint

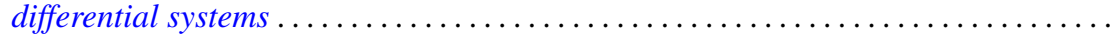

Ronald Fintushel, Local $S^{1}$ actions on 3-manifolds .

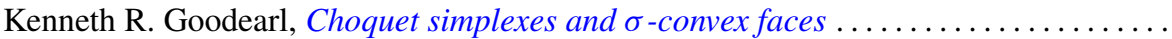

John R. Graef, Some nonoscillation criteria for higher order nonlinear differential

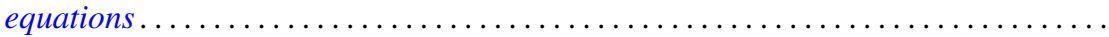

Charles Henry Heiberg, Norms of powers of absolutely convergent Fourier series: an example.

Les Andrew Karlovitz, Existence of fixed points of nonexpansive mappings in a space

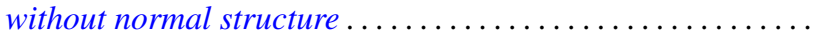

Gangaram S. Ladde, Systems of functional differential inequalities and functional

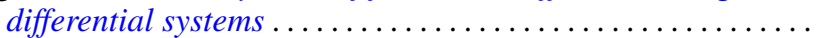

Joseph Michael Lambert, Conditions for simultaneous approximation and interpolation with norm preservation in $C[a, b]$.

Ernest Paul Lane, Insertion of a continuous function.

Robert F. Lax, Weierstrass points of products of Riemann surfaces .

Dan McCord, An estimate of the Nielsen number and an example concerning the Lefschetz fixed point theorem...

Paul Milnes and John Sydney Pym, Counterexample in the theory of continuous functions on topological groups...

Peter Johanna I. M. De Paepe, Homomorphism spaces of algebras of holomorphic functions

Judith Ann Palagallo, A representation of additive functionals on $L^{p}$-spaces,

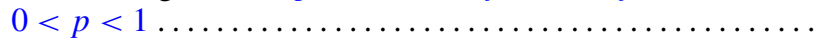

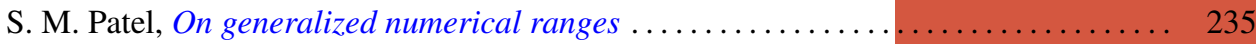

Thomas Thornton Read, A limit-point criterion for expressions with oscillatory

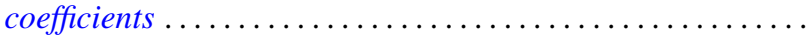

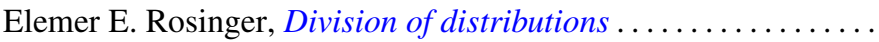

Peter S. Shoenfeld, Highly proximal and generalized almost finite

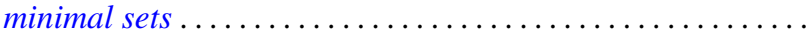

R. Sirois-Dumais and Stephen Willard, Quotient-universal sequential spaces

Robert Charles Thompson, Convex and concave functions of singular values of matrix sums....

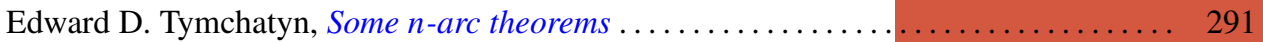

